\title{
Radial Ball Bearing Inner Race Defect Width Measurement using Analytical Wavelet Transform of Acoustic and Vibration Signal
}

\author{
Dibya Prakash Jena ${ }^{1}$, Manpreet Singh ${ }^{2}$, Rajesh Kumar ${ }^{2}$ \\ ${ }^{1}$ Manufacturing Management, Birla Institute of Technology Pilani, Rajasthan-333031, INDIA \\ ${ }^{2}$ Precision Metrology Laboratory, Department of Mechanical Engineering \\ Sant Longowal Institute of Engineering and Technology, Longowal - 148106, INDIA
}

\begin{abstract}
In the present work, an experiment is carried out with a customized test setup where the seeded defects are introduced in the form of an axial groove on the inner race of a radial ball bearing. The nature of the acoustic and vibration signal bursts, and their correlation with the inner race defects, are established and estimated. Experimental investigation reveals that the analytical wavelet transform (AWT) is an effective tool for analyzing the acoustic and vibration signals, transmitted from the bearing, in order to characterize and measure the defect size. In the recent work, AWT followed by the time marginal integration (TMI) have been implemented on acoustic and vibration signals of a defective radial bearing. Size of the defect in the inner race of bearing is corroborated well with AWT scalogram. The segregation of the defect is carried out on TMI graph across the highest amplitude spike, which is due to signal burst (due to a contact of ball with bearing inner race defect). This manual demarcation on TMI graph in time axis provides the time duration (contact between a ball and the inner race defect). Using this time duration of the ball passed over bearing inner race defect, RPM of shaft mounted across bearing, and the fundamental train frequency, the defect width is estimated. The deviation of the measured width from the actual, using the proposed method, is sought below $5 \%$. Summarizing, the proposed method can be reckoned a suitable and reliable measurement of radial bearing inner race defect width from acoustic and vibration signal.
\end{abstract}

Keywords: Condition Monitoring, Analytical Wavelet Transform, Scalogram, Time Marginal Integration (TMI)

\section{INTRODUCTION}

$\mathrm{F}_{\mathrm{a} a \mathrm{i}}$ AULT DETECTION of the rolling elements, i.e. gear and bearing faults, has been gaining importance in recent years because of its detrimental influence on the reliability of the machines. Vibration signature analysis is the most commonly used fault-detection technique employed in rotor- bearing systems. Detection of the fault and its severity are two important steps or features of a condition monitoring system. Lifetime of a machine component is determined by the severity of the fault. It is crucial, especially in critical systems, where continual operation is generally indispensable. The bearing defects can be either distributed or local type or combination of both. The distributed defects can be the surface roughness, waviness, misaligned races, and off-size rolling elements. Localized defects are developed in the raceways, rollers and cage of a bearing. The periodic impacts occur at ball-passing frequency (characteristic defect frequencies), which can be estimated from the bearing geometry and the rotational speed. Sometimes the defect frequencies are not observable with the help of the Fast Fourier Transform (FFT) spectrum, because the impulses generated by the defects are masked by noise. To overcome this problem, signal processing techniques are implemented by many researchers. Several methods, based on vibration and acoustic signal processing, have been developed to detect bearing local faults. Recently, the application of the wavelet transform has emerged in the context of bearing fault detection and condition monitoring. Wavelet transform has the local characteristic of timedomain as well as frequency domain and its adaptive timefrequency window.
During the last few years a significant progress, in the capabilities of acoustic instrumentation together with the signal processing technique, has made it possible to extract the useful diagnostic information from the acoustic signals. Investigators reported that the acoustic emission (AE) parameters can identify the bearing defects before they appear in the vibration acceleration range [1,2]. The variations in standard $\mathrm{AE}$ statistical parameters can also be used in bearing fault diagnosis. Apart from statistical analysis, there are many analytical techniques established, such as resonance demodulation, instantaneous power spectrum distribution and conditional moment analysis, etc., which have been used for processing of the vibration signals to obtain useful diagnostic information $[3,4]$. In case of highly transient signals such as $\mathrm{AE}$, the time and frequency components are very much dependent on each other. This has prompted many researchers to work on automatic defect identification using wavelet algorithm [5,6]. Abdullah et al. have reported the relationship between AE RMS amplitude and kurtosis for a range of defect conditions and the relationship between the defect size and the duration of $\mathrm{AE}$ burst [7]. They have also concluded that these statistical parameters are more sensitive to the onset and growth of defects over vibration measurements.

Measured acoustic signal is often severely tainted by various noises such as the background noise present due to the instrumentation or circuitry of the measuring device as well as the vibrations generated by other mechanical components. An effective denoising method is necessary to remove such noise and interferences. Two major denoising approaches, namely the wavelet threshold-based (also 
known as decomposition-based) and the wavelet filter-based approach, [3] have been used to filter the vibration signals measured from faulty bearing or gear systems. Bozchalloi and Liang have reported that the reliable condition monitoring would not be possible without proper denoising [8]. The investigation report says the performance of the wavelet based denoising can be improved considerably by preprocessing of the signal using spectral subtraction. However, Al-Dossary et al. have investigated changes in AE waveform for varying seeded defect sizes in a radially loaded rolling element bearing including the correlation of the defect size to the AE parameters [9]. The relationship between the duration of AE transient bursts associated with seeded defects (the actual geometric size of the defect) has been established by them. An increase in the defect size results in an increase in levels of the AE energy for outer and inner race seeded defects. One of their major conclusions is that, for inner race and outer race defect cases, the time duration between successive AE transients is not always identical. This variation is attributed to slip of the cage assembly. These observations also offer an explanation for mismatch of the number of $\mathrm{AE}$ transients with the theoretical defect frequency. Prabhakar et al. have investigated the bearing faults using discrete wavelet transform (DWT). The impulses in vibration signals due to faults are prominent in wavelet decompositions and bursts in signal appear periodically with a time period corresponding to characteristic defect frequencies [10]. Junsheng et al. have investigated two methods, namely the scale wavelet power spectrum comparison and the auto-correlation analysis of time-wavelet power spectrum, to find the characteristic of roller bearing fault from vibration signals. The impulse response wavelet is constructed by using the continuous wavelet transform (CWT), which helps in extracting the feature of the seeded faults [11]. Du and Yang have introduced the empirical mode decomposition (EMD) method and suggested an improvement in computation of local mean by using the averaging of the successive extrema. They substituted conventional envelope mean by the average mean and applied the improved EMD method in the vibration analysis of ball bearings and observed the suitability over discrete wavelet decomposition [16]. Rai and Mohanty have investigated bearing fault using FFT of intrinsic mode functions of Hilbert-Huang transform (HHT). The Hilbert-Huang Transform (HHT) technique provides multi-resolution in various frequency scales and takes the signal's frequency content and their variation into consideration. HHT analyses the vibration signal using intrinsic mode functions (IMFs), which are extracted using the process of empirical mode decomposition (EMD) [12]. Karacay and Akturk have reported the formation and development of localized defects in ball bearings by using a new bearing through to failure using statistical and spectral methods. They have observed that the traditional values such as peak-to-peak value, RMS, Crest factor and kurtosis show damage at the ball bearing but do not give information about the location of defect. They conduct spectrum analysis at specified test duration in order to predict defect locations [13]. Stein et al. have demonstrated the compact vibration measurement system for condition monitoring purpose, which also has been implemented to develop a real-time monitoring system for the current experiment [14]. Another aspect of embedded DSP system integration using independent component analysis (ICA) can be also used for real-time fault diagnosis [15]. Recently Manpreet and Rajesh have implemented a wavelet based technique to process the contaminated acoustic signal and diagnose defect in the rotating element. The acoustic emissions from cage ball bearing with missing balls at different positions are recorded with the help of a microphone using Daubechies wavelet [5]. They have observed that the wavelet based technique is very effective in the diagnosis of faults in a radial ball bearing. Compared to the healthy bearing, there is a sudden decrease in acoustic emission when missing ball position comes in front of the microphone during rotation. Position of the missing ball and rotation of the shaft on which the bearing is mounted can be easily estimated by analyzing the decomposed signal. But any of the methods developed based on denoising, however, cannot provide accurate results, as by denoising the signal smoothens and in the process some of the useful information gets filtered out.

In the current work the acoustic and vibration signals of the radial bearing having inner race defect have been processed with a signal processing technique to measure the defect width. The defect is introduced in the form of an axial groove in the inner race of the bearing. The Analytical Wavelet Transform (AWT) followed by Time Marginal Integration (TMI) has been investigated on acoustic and vibration signals to identify the presence and severity of defect. AWT scalogram, which is a joint-time-frequency representation, is used to measure the defect width. It retains most of the basic features of the Fourier transform and also captures temporal and spectral characteristics of the acoustic and vibration signal in much more detail. TMI returns the result of integrating the AWT spectrogram along the frequency axis. The discrimination was carried out on the TMI graph across the highest amplitude spike which is due to signal burst due to the contact of ball and bearing inner race defect. This manual differentiation on the TMI graph on the time axis will provide the time duration of ball in contact with inner race defect width. Using the time duration of ball passed over bearing inner race defect, RPM of the shaft mounted across bearing and fundamental train frequency, the defect width has been estimated.

\section{THEORY}

\section{A. Characteristic of Rolling Element Defect Frequencies and Measuring Parameters}

It is a well-known fact that local defects or wear cause periodic impulses in acoustic emission and vibration signals. The amplitude and period of these impulses are determined by shaft rotational speed, fault location, and bearing dimensions. The frequencies of these impulses, considering different defect locations, can be calculated from the wellestablished relations $[2,10]$. If the shaft rotational frequency per second is $F_{s}$, the number of balls/rollers in the bearing is ' $\mathrm{Z}$ ', the ball/roller diameter is ' $\mathrm{d}$ ', the pitch diameter of the bearing is ' $\mathrm{D}$ ', and the contact angle is ' $\alpha$ ' ( $\alpha=0$ for a radial bearing), then the rotational frequency of a rolling 
element, $\mathrm{F}_{\mathrm{re}}$ and the rotational frequency of the ball cage with a stationary outer race, $\mathrm{F}_{\mathrm{bcsor}}$ are expressed as

$$
\begin{aligned}
& \mathrm{F}_{\mathrm{re}}=\left[\mathrm{F}_{\mathrm{s}} / 2\right][\mathrm{D} / \mathrm{d}]\left[1-(\mathrm{d} / \mathrm{D})^{2} \cos ^{2} \alpha\right] \\
& \text { and } \mathrm{F}_{\mathrm{bcsor}}=\left[\mathrm{F}_{\mathrm{s}} / 2\right][1-(\mathrm{d} / \mathrm{D}) \cos \alpha]
\end{aligned}
$$

The frequency at which the rolling element contacts a fixed point on a rotating inner race with a stationary outer race is expressed as:

$$
\mathrm{F}_{\text {recri }}=\left[\mathrm{ZF}_{\mathrm{s}}\right][1-0.5\{1-(\mathrm{d} / \mathrm{D}) \cos \alpha\}]
$$

The bearing health can be monitored by the statistical parameters such as kurtosis, octave, etc. of the acoustic and vibration signal. The crest factor corresponds to the ratio between the crest value (maximum absolute value reached by the function representative of the signal during the considered period of time) and the RMS value (efficient value) of the signal and can be expressed as [11]

$$
\text { Crest Factor }=\frac{\text { CrestValue }}{\text { R.M.S.value }}=\frac{\sup |x(n)|}{\sqrt{(1 / N) \sum_{n=1}^{N}[x(n)]^{2}}},
$$

where ' $\mathrm{N}$ ' is the number of samples taken within the time domain signal $\mathrm{x}(\mathrm{n})$. Kurtosis is a measure of the peak and valley in a signal. Kurtosis is a statistical parameter to analyze the distribution of the vibratory amplitudes contained in a time domain signal and is often used as an indicator of impulsiveness. With $\mathrm{M}_{4}$ the fourth order statistic moment, $\mathrm{M}_{2}$ the second order statistic moment, $\mathrm{x}(\mathrm{n})$ the amplitude of the signal for the $\mathrm{n}$ sample, $\tilde{\mathrm{x}}$ the mean value of the amplitudes, the kurtosis factor can be expressed in the following manner [11]

$$
\text { Kurtosis }=\frac{M_{4}}{M_{2}^{2}}=\frac{(1 / N) \sum_{n=1}^{N}(x(n)-\widetilde{x})^{4}}{\left[(1 / N) \sum_{n=1}^{N}(x(n)-\widetilde{x})^{2}\right]^{2}}
$$

In the context of machine fault detection, a high kurtosis value is treated as a sign of the presence of faults in a rotating mechanical system [8].

\section{B. Analytical Wavelet Transform and Time Marginal Integration}

Digital signal processing is widely implemented for fault diagnosis and condition monitoring. Among such processing methods, Fourier analysis and Short Term Fourier Transform (STFT) resolve the time domain waveform into its sinusoidal components by taking a block of time-domain data and returning it to the frequency spectrum. Thus, the Fourier transform does not yield a continuous spectrum. Instead, it returns a discrete spectrum where the frequency content of the waveform is resolved into a finite number of frequency lines, or bins. The elementary analysis has been done with Fourier transform. But a time-frequency (T-F) analysis is desired for such transient signal analysis. The T$\mathrm{F}$ analysis has to be used properly to characterize highly transient events such as accelerations of impact tools, because their spectral characteristics change rapidly in time. In fact, the time and frequency are not separate, but interwoven concepts. The wavelet transform is a T-F signal analysis technique which uses variable scales to satisfy both time and frequency resolution requirements more precisely.

In 1982, Jean Morlet, a French geophysical engineer, discovered the idea of the wavelet transform. Morlet has first introduced the idea of wavelets as a family of functions constructed from translations and dilatations of a single function called the "mother wavelet". Many researchers (Grossmann, Meyer, Mallat, Daubechies, etc.) have developed and enhanced this new signal-processing tool to make it the most efficient in the structural health-monitoring field. The wavelet transform can be thought of as an extension of the classic Fourier transform, except that, instead of working on single scale (time or frequency), it works on a multi-scale basis. The wavelet transform can be classified as continuous or discrete [18]. The main advantage of the continuous wavelet transformation (CWT) is its ability to provide information simultaneously in time and scale with adaptive windows. The CWT offers promising tools for the estimation of modal parameters and new perspectives for damage identification within the structures. Calculating wavelet coefficients at every possible scale is a fair amount of work, and it generates an awful lot of data.

The analytical wavelet transform (AWT), a continuous wavelet transform, can be viewed as a hybrid of the wavelet transform and Fourier transform. The AWT characterizes a signal by its frequency and amplitude as in the Fourier transform. However, the AWT is a function of time, unlike the Fourier transform. The AWT is an ideal tool for the analysis of transient events in the vibration and acoustic signals.

Mathematically, the AWT of a signal $\mathrm{a}_{\mathrm{x}}(\mathrm{t})$ is $\mathrm{W}_{\mathrm{s}} \mathrm{a}_{\mathrm{x}}(\mathrm{t})$, and can be defined as:

$$
\begin{gathered}
\mathrm{W}_{\mathrm{s}} \mathrm{a}_{\mathrm{x}}(\mathrm{t})=\int_{-\infty}^{+\infty} \mathrm{a}_{\mathrm{x}}(\mathrm{u}) \psi_{\mathrm{s}}^{*}(\mathrm{u}-\mathrm{t}) \mathrm{dt} \\
=\int_{-\infty}^{+\infty}(1 / \mathrm{s}) \mathrm{a}_{\mathrm{x}}(\mathrm{u}) \mathrm{g}\left(\frac{\mathrm{u}-\mathrm{t}}{\mathrm{s}}\right) \mathrm{e}^{-\mathrm{j} \eta(\mathrm{u}-\mathrm{t} / \mathrm{s})} \mathrm{dt}
\end{gathered}
$$

where $\mathrm{s}$ is the scale, $\psi_{\mathrm{s}}=\mathrm{g}(\mathrm{u} / \mathrm{s}) \mathrm{e}^{\mathrm{j} \eta(\mathrm{u} / \mathrm{s})}$ is the wavelet function of scale $\mathrm{s}, \mathrm{j}=\sqrt{-1}$ and $\eta$ is a parameter that relates the scale with the frequency [17].

The AWT is a wavelet transform that retains most of the basic features of the Fourier transform but captures the temporal and spectral characteristics of the acoustic and vibration signal in much more detail. We have implemented AWT with complex Morlet wavelet [3]. The Morlet wavelet can be expressed as:

$$
\varphi_{0}(\mathrm{t})=\left(-1 / \sqrt[4]{\sigma^{2} \pi}\right) \exp \left(-\left(\mathrm{t}^{2} / 2 \sigma^{2}\right)+j \omega_{0} t\right),
$$


where ' $\sigma$ ' is the standard deviation of Gaussian envelope of the Morlet wavelet, and ' $\omega_{0}$ ' is the central frequency of mother wavelet (which is $3 \pi / 2$ in our analysis). The center frequency, $\omega_{0} / 2$ of the scaled wavelet is inversely proportional to scale, ' $a$ '.

For effective analysis of the AWT spectrum, the time marginal integral (TMI) is carried out. The TMI returns the result of integrating the spectrogram along the frequency axis, or the integral of each row of spectrogram. The TMI spectrum is equivalent to the smoothed instantaneous power of the signal. The instantaneous power reveals how the power of the signal changes over time [3]. The equation below defines the time marginal integration:

$$
\mathrm{TMI}=\int_{-\infty}^{+\infty} \mathrm{SP}(\mathrm{t}, \omega) \mathrm{d} \omega
$$

where $\operatorname{SP}(t, \omega)$ is the spectrogram of the signal in time frequency plane.

\section{EXPERIMENT}

The experiments are conducted to diagnose the inner race defect in a radial ball bearing (no. 6206) and measure width of the defect. The internal diameter of the ball bearing is 30 $\mathrm{mm}$, external diameter $62 \mathrm{~mm}$, and total number of balls 9 . The bearing is mounted on the shaft which is rotated by a 200 Watt motor. An accelerometer is mounted on the top of the bearing bracket using the thread screw mechanism and the microphone is placed near the bearing housing. Schematic of experimental setup is shown in Fig.1. The shaft connected to the motor is rotated at $1500 \mathrm{rpm}$. After stabilizing the rotation for 10 minutes, the accelerometer signal from the bearing assembly is recorded using SCXI$1530^{\circ}$ data acquisition system and the microphone signal is recorded by sound card with a customized software application developed in LabVIEW ${ }^{\odot} 7.1$ environment. In the first phase of the experiment, the acoustic signal and vibration signal are captured on healthy bearing at $20 \mathrm{~K}$ and $44.1 \mathrm{~K}$ samples per second, respectively. Microphone and accelerometer signals are acquired in terms of voltage (volt) and the recorded signals are processed in LabVIEW. The signals are processed offline (of 0.5 second duration). A typical recording of microphone and accelerometer signal of time duration 0.5 seconds for the healthy bearing is shown in Fig.2(a) and Fig.(b), respectively. As observed from the Fourier transform the prominent frequencies in the acoustic and vibration signal are in the range of $25-250 \mathrm{~Hz}$.

In the second phase of the experiment, the defective bearing is mounted in the test setup. A groove defect was introduced on the inner race of the bearing in the axial direction with the help of sharp rotating cutter disc of $2 \mathrm{~mm}$ thickness. The width of grove is $2.1 \mathrm{~mm}$ and depth of the groove is $1 \mathrm{~mm}$. A typical recording of the microphone and accelerometer signal for the same time duration, i.e. 0.5 seconds, is shown in Fig.3(a) and 4(a), respectively. The FFT spectrum of acoustic signal and vibration signal is shown in Fig.3(b) and 4(b), respectively. One can notice that the amplitude of the acoustic and vibration signal of defective bearing is much higher than that of the healthy bearing. In FFT of acoustic signal the prominent frequencies in the signal are $24.5 \mathrm{~Hz}, 98 \mathrm{~Hz}, 200 \mathrm{~Hz}$ and $250 \mathrm{~Hz}$ in the band of 0 to $250 \mathrm{~Hz}$. It has also been observed that due to defect, high frequency components also became prominent in $500-2500 \mathrm{~Hz}$. In FFT of vibration signal, the prominent frequencies are in the range of $15-3000 \mathrm{~Hz}$. It has also been observed that due to the defect, high frequency components also became prominent.

The defect (groove) on the inner race of the bearing results in sudden increase in amplitude of the signal.

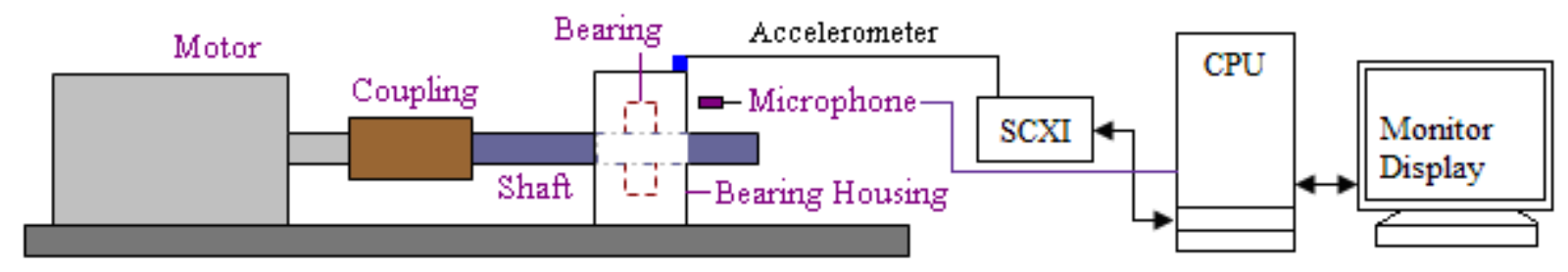

Fig.1. Schematic of Experimental Setup

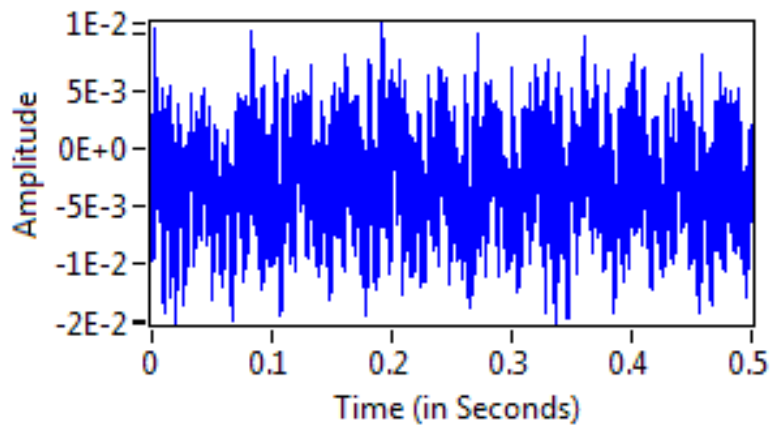

(a)

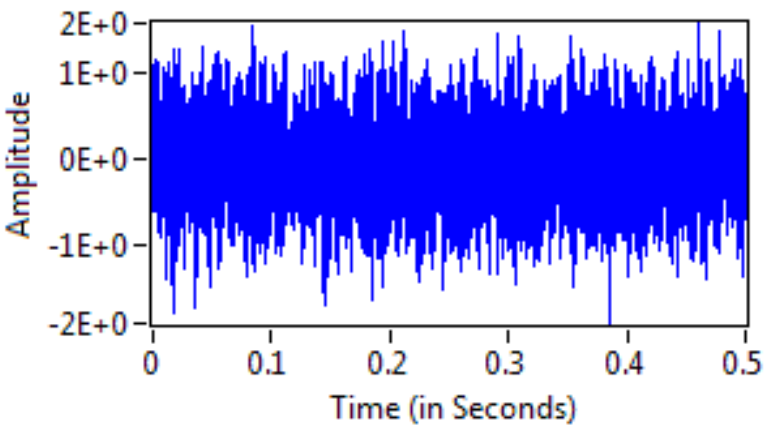

(b)

Fig.2. Healthy bearing acoustic signal (a) and vibration signal (b) 


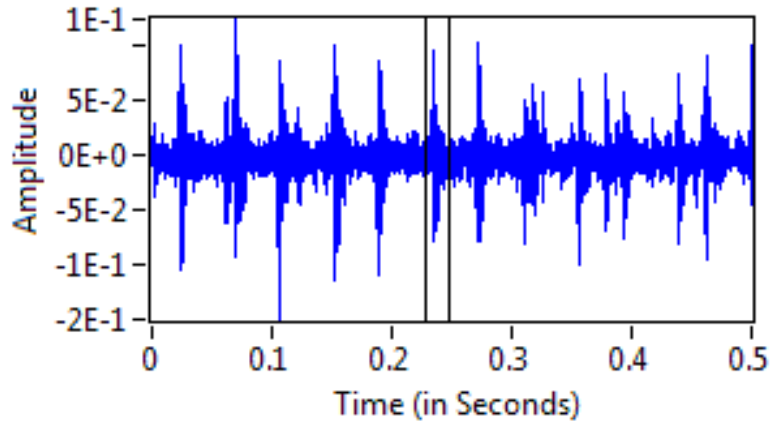

(a)

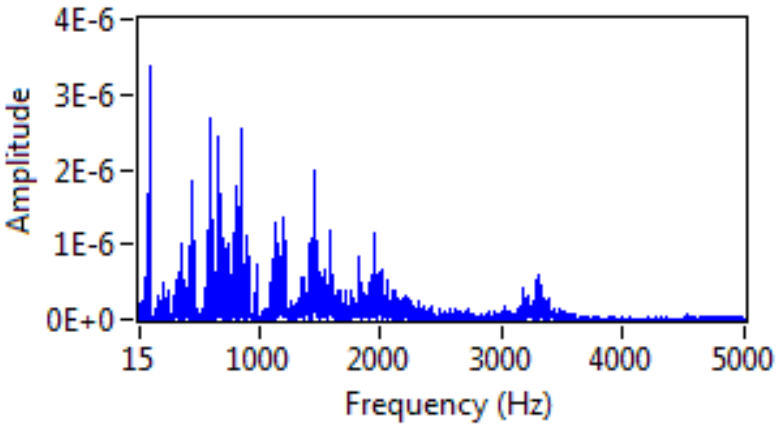

(b)

Fig.3. Defective bearing acoustic signal (a) and FFT of the signal (b)

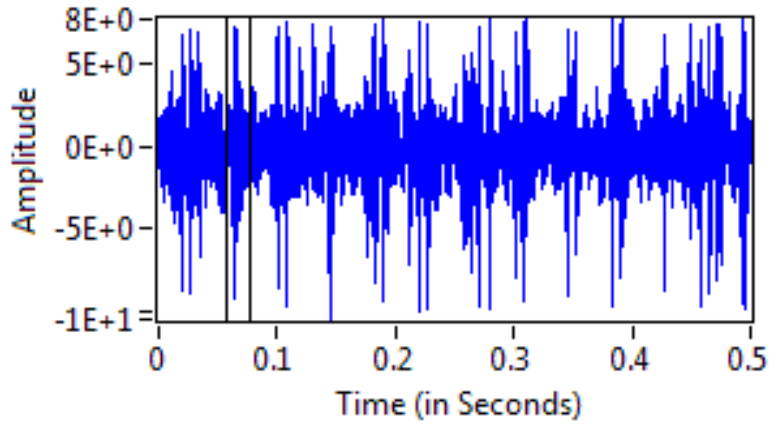

(a)

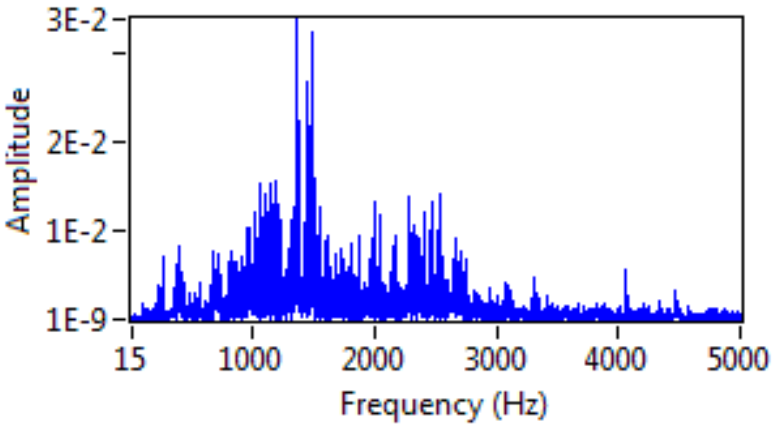

(b)

Fig.4. Defective bearing vibration signal (a) and FFT of the signal (b)

\section{DISCUSSION}

The statistical quantities such as RMS value, standard deviation (SD), skewness, signal to noise ratio (SNR) and kurtosis for the acoustic signals shown in Fig.2(a) (healthy bearing) and Fig.3(a) (defective bearing) and vibration signals shown in Fig.2(b) (healthy bearing) and Fig.4(a) (defective bearing) are calculated and given in Table1 and Table2, respectively. The SNR is the inverse of the coefficient of variation $\left(\mathrm{C}_{\mathrm{v}}\right)$, i.e. $\mathrm{SNR}=1 / \mathrm{C}_{\mathrm{v}}$ and $\mathrm{C}_{\mathrm{v}}=\sigma /|\mu|$. The absolute value is taken for the mean to ensure $\mathrm{C}_{\mathrm{v}}$ is always positive. From Table 1 and Table 2 it is observed that defective bearing has higher RMS, SD, kurtosis, and crest factor but has lower skewness and SNR than the healthy bearing. Defect on the inner race of the bearing results in sudden increase in amplitude of the signal.
The ratio of RMS value of amplitude in a part of the signal under consideration to the RMS value of noise in the signal can give an idea about the burst. For the healthy bearing, this ratio should be close to 1 . For the data from defective bearing having groove, during the burst, average value of this ratio comes to be 2.933 for acoustic signal and 2.576 for vibration signal. Moreover, the defect due to groove can be corroborated from the burst.

Subsequently, to analyze the defect width, we have minutely observed the nature of the acoustic signal during burst. When the ball passes over the uneven surface, complex high frequency acoustic signals are generated and transmitted. A portion of the signal marked between time $0.228 \mathrm{sec}$. to $0.248 \mathrm{sec}$ in Fig.3(a) is extracted and shown in Fig.5(a) (I).

Table 1. Statistical estimates of the acoustic signals

\begin{tabular}{|l|l|l|l|l|l|l|}
\hline Bearing condition & RMS & Std. Dev. & Skewness & SNR & Kurtosis & Crest Factor \\
\hline Healthy & 0.00472 & 0.00369 & 0.03594 & 1.04169 & 2.96143 & 4.09005 \\
\hline Groove Defect & 0.01445 & 0.01415 & -0.08758 & 0.69858 & 10.87315 & 14.02610 \\
\hline
\end{tabular}

Table 2. Statistical estimates of the vibration signals

\begin{tabular}{|l|l|l|l|l|l|l|}
\hline Bearing condition & RMS & Std. Dev. & Skewness & SNR & Kurtosis & Crest Factor \\
\hline Healthy & 0.44629 & 0.44509 & -0.00946 & 0.79895 & 3.05191 & 4.23012 \\
\hline Groove Defect & 1.83710 & 1.83641 & -0.18682 & 0.68627 & 7.77667 & 7.87534 \\
\hline
\end{tabular}



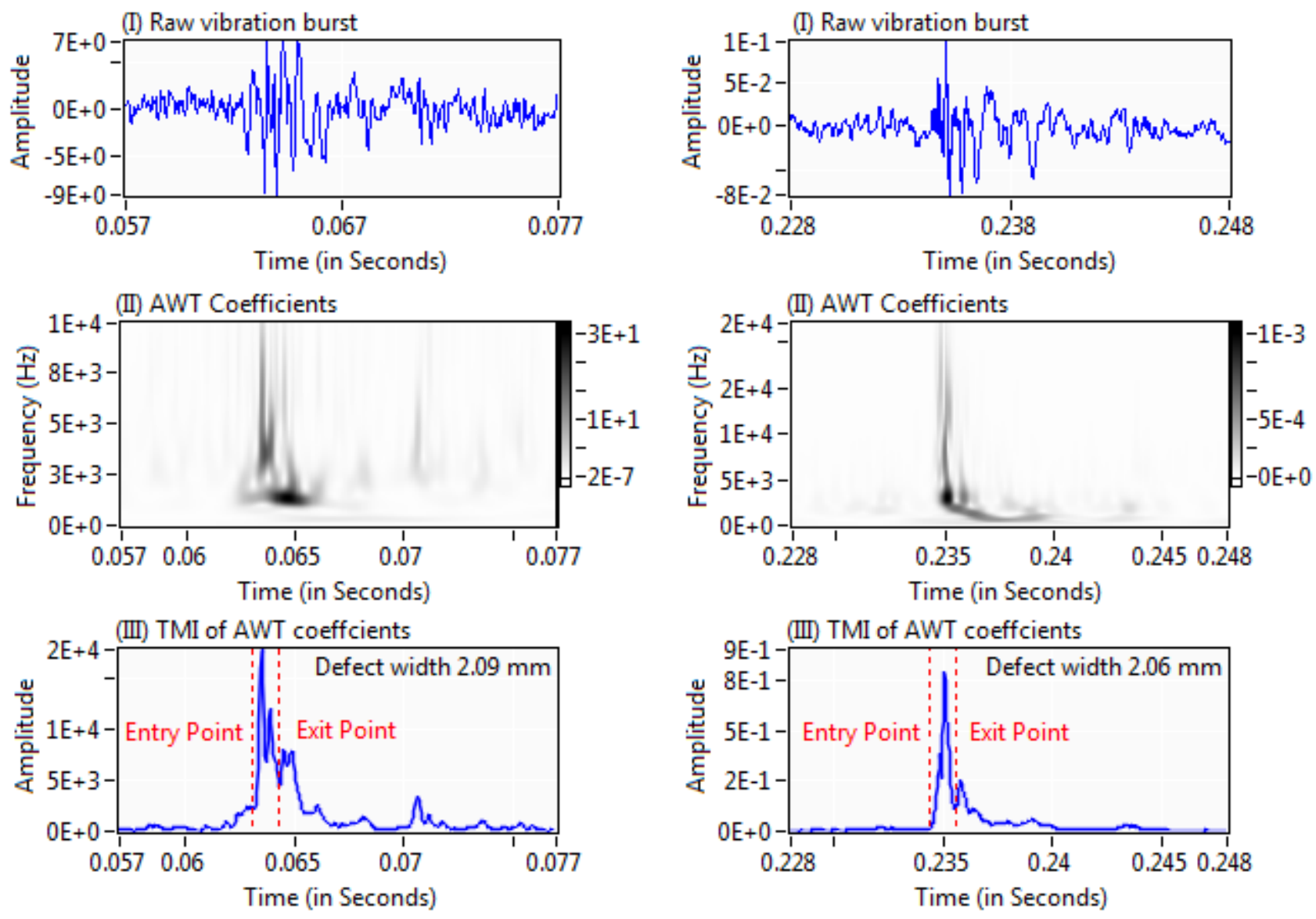

(a)

(b)

Fig.5. Single acoustic burst analysis (a) and vibration burst analysis (b); (I) raw signal burst, (II) AWT spectrum, (III) TMI of AWT spectrum

The AWT is implemented on the extracted signal at scale 2048 with even frequencies and energy normalization method. The resultant scalogram in terms of intensity is shown in Fig.5(a) (II) which is a kind of joint-timefrequency representation with an adaptive time-frequency resolution. The $x$-axis represents time, and the $y$-axis represents the frequency. The color (in color scalogram) indicates the square magnitude or the phase of the signal. Large scales correspond to low frequency and small scales correspond to high frequencies. Width of the groove in terms of time duration of the high frequency components is observed like a droplet on the scalogram between time 0.234 sec and $0.235 \mathrm{sec}$.

The TMI graph shows a major spike during the same duration. The manual discrimination is carried out to find the time duration $\Delta t$ of major spike due to burst, shown in Fig.5(a) (III). The defect size present on bearing inner race is calculated by the use of the acoustic burst duration $\Delta \mathrm{t}$ from experimental analysis, fundamental train frequency (FTF) and average outer diameter of inner race $\mathrm{D}_{\mathrm{IR}}$, which can be expressed as mentioned in the equation below.

Defect width in inner race:

$$
\mathrm{L}_{\mathrm{id}}=\pi \times \Delta \mathrm{t} \times \mathrm{D}_{\mathrm{IR}} \times \mathrm{FTF},[\text { millimeter }]
$$

From the data of ten different acoustic bursts, average size of the groove defect in the bearing was evaluated to be 2.09 $\mathrm{mm}$ with variance of $0.05 \%$ and standard deviation of 0.04 $\mathrm{mm}$. Physical measurement of groove width was also done and came to be $2.1 \mathrm{~mm}$. Error in measurement of groove width obtained from signal processing using AWT followed by TMI and physical measurement is less than $5 \%$.

Similarly, a portion of the vibration signal marked between time $0.057 \mathrm{sec}$. to $0.077 \mathrm{sec}$ in Fig.4(a) is extracted and shown in Fig.5(b) (I). Similar to what was mentioned above, the AWT is implemented on the extracted signal at scale 2048 with even frequencies and energy normalization method. The resultant scalogram in terms of intensity is shown in Fig.5(b) (II). Width of groove in terms of time duration of the high frequency components is observed like a droplet on the scalogram between time $0.063 \mathrm{sec}$ and $0.064 \mathrm{sec}$. The TMI graph shows a major spike during the same duration. The manual discrimination is carried out to find the time duration $\Delta \mathrm{t}$ of the major spike due to burst, shown in Fig.5(b) (III). The defect size present on bearing inner race is calculated by using equation (9). From the data of ten different vibration bursts, the average size of the groove defect in the bearing is evaluated and comes to be $2.09 \mathrm{~mm}$ with standard deviation of $0.0195 \mathrm{~mm}$ and variance of $0.038 \%$. Error in measurement of the defect 
width obtained from vibration signal processing using AWT followed by TMI and physical measurement is less than $5 \%$.

To see the effect of wavelet denoising, on the signal of bearing with groove defect, we apply the discrete wavelet decomposition method. The signal is decomposed using $\mathrm{db} 04$ wavelet up to level-2 and soft thresholding. It is observed that clarity in signal on amplitude versus time scale improved but at the same time the nature of burst changed. The information about the defect size from AWT was lost as the information got filtered out during denoising. Results show that information of inner race bearing defects can be preserved if the raw/original signal is processed for AWT rather than the processing of denoised signal by AWT.

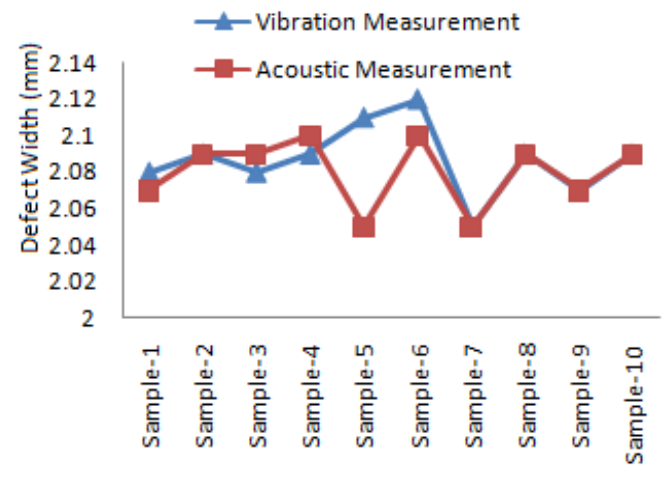

Fig.6. Defect width measurement of multiple samples

Further research on the proposed work can be carried out in two ways. The first one is the numerical simulation using ANSYS transient vibration analysis and contact analysis. The second one can be pursued in the area of automatic defect localization and classification $[1,4]$. In contest, further research is also initiated by the author. This information is provided to understand the wide nature of scope of this particular problem. However, signal processing of acoustic and vibration signals for fault detection and measurement is presented in the current research.

\section{CONCLUSION}

The acoustic and vibration signal of defective bearing has higher RMS, SD, kurtosis, and crest factor but has lower skewness and SNR than the healthy bearing which gave indication about the presence and severity of defect. The denoising technique is helpful in spotting the defect but is not suitable for evaluating the size and nature of defect. Experimental investigations reveal that AWT followed by TMI is an effective tool in analyzing the acoustic and vibration signal transmitted from radial bearing in order to characterize and find out the size of the defect. Size of defect in the bearing can be corroborated well with the AWT scalogram. Error in measurement obtained from signal processing by AWT and physical measurement is less than 5\%. Summarizing, the proposed method can be reckoned in precise radial bearing inner race defect measurement from acoustic and vibration signal processing.

\section{ACKNOWLEDGMENT}

Dibya Prakash Jena is thankful to the management of Robert Bosch Engineering and Business Solutions, Bangalore for permitting him to carry out his MS from Birla Institute of Technology \& Science, Pilani under OffCampus Work-Integrated Learning Programs with Dr. Rajesh Kumar as his mentor.

\section{REFERENCES}

[1] Orhan, S., Akturk, N., Celik, V. (2006). Vibration monitoring for defect diagnosis of rolling element bearings as a predictive maintenance tool: Comprehensive case studies. NDT\&E International, 39, 293-298.

[2] Zarei, J., Poshtan, J. (2009). An advanced Park's vectors approach for bearing fault detection. Tribology International, 42, 213-219.

[3] National Instruments. (2007). LabVIEW - Advance Digital Signal Processing User Manual.

[4] Ban, J.E., Rho, B.H., Kim, K.W. (2007). A study on the sound of roller bearings operating under radial load. Tribology International, 40, 21-28.

[5] Kumar, R., Jena, D.P., Bains, M. (2010). Identification of inner race defect in radial ball bearing using acoustic emission and wavelet analysis. In ISMA 2010 : International Conference on Noise and Vibration Engineering, 20-22 September 2010. Leuven, Belgium, 2883-2891.

[6] Messer, S.R., Agzarian, J., Abbott, D. (2011). Optimal wavelet denoising for phonocardiograms. Microelectronics Journal, 32, 931-941.

[7] Al-Ghamd, M., Mba, D. (2006). A comparative experimental study on the use of acoustic emission and vibration analysis for bearing defect identification and estimation of defect size. Mechanical Systems and Signal Processing, 20, 1537-1571.

[8] Bozchalooi, S., Liang, M. (2008). A joint resonance frequency estimation and in-band noise reduction method for enhancing the detectability of bearing fault signals. Mechanical Systems and Signal Processing, 22, 915-933.

[9] Al-Dossary, S., Raja Hamzah, R.I., Mba, D. (2009). Observations of changes in acoustic emission waveform for varying seeded defect sizes in a rolling element bearing. Applied Acoustics, 70, 58-81.

[10] Prabhakar, S., Mohanty, A.R., Sekhar, A.S. (2002). Application of discrete wavelet transform for detection of ball bearing race faults. Tribology International, 35, 793-800.

[11] Junsheng, Ch., Dejie, Y., Yu, Y. (2007). Application of an impulse response wavelet to fault diagnosis of rolling bearings. Mechanical Systems and Signal Processing, 21, 920-929.

[12] Rai, V.K., Mohanty, A.R. (2007). Bearing fault diagnosis using FFT of intrinsic mode functions in Hilbert-Huang transform. Mechanical Systems and Signal Processing, 21, 2607-2615. 
[13] Karacay, T., Akturk, N. (2009). Experimental diagnostics of ball bearings using statistical and spectral methods. Tribology International, 42, 836-843.

[14] Stein, G.J., Chmúrny, R., Rosík, V. (2011). Compact vibration measuring system for in-vehicle applications. Measurement Science Review, 11 (5), 154-159.

[15] Patil, D., Das, N., Routray, A. (2011). Implementation of Fast-ICA: A performance based comparison between floating point and fixed point DSP platform. Measurement Science Review, 11 (4), 118-124.

[16] Du, Q., Yang S. (2007). Application of the EMD method in the vibration analysis of ball bearings. Mechanical Systems and Signal Processing, 21, 2634-2644.
[17] Kim, J., Welcome, D.E., Dong, R.G., Song, W.J., Hayden, Ch. (2007). Time-frequency characterization of hand-transmitted, impulsive vibrations using analytic wavelet transform. Journal of Sound and Vibration, 308, 98-111.

[18] Khorrami, H., Moavenian, M. (2010). A comparative study of DWT, CWT and DCT transformations in ECG arrhythmias classification. Expert Systems with Applications, 37, 5751-5757.

Received September 19, 2011. Accepted July 23, 2012. 